\title{
Reliability Analysis of UAV Aviation Electronic System Based on FMEA Han-Ning ${ }^{1, a}$, Wu Guoqing ${ }^{2, b}$, Xia-Mingfei ${ }^{3, c}$, Wang xiaocong ${ }^{4, d}$ Machine technology research Institute Machine Engineering College, Shijiazhuang, 050000, China \\ aemail: oec_ljw2009@163.com
}

Keywords: aviation electronic sub-system; FMEA; reliabitly; risk prilrity number

\begin{abstract}
In order to improve the reliability of aviation electronic sub-system in UAV, the analysis method based on Failure Mode and Effects Analysis (FMEA) is put forward. The risk factors and correction measurement feasibility parameters are quantified with fuzzy theory. Then the fault modes with higher risk priority number (RPN) based on Scree-Plot method are verfied. Finally, as the ratio of RPN alteration and correction parameter F chosen for the basis of choosing correction methods, the correction method with the highest ratio is chosen to be used to maintain the UAV aviation electronic sub-system. The real example demonstrates the validity of this method.
\end{abstract}

\section{Introduction}

UAV aviation electronic system is one of the most important systems in UAV control system. Compared with other systems, it characterizes as more electronic circuits, more severe effects when becoming failure.

Failure Modes and Effect Analysis (FMEA) is one method used to analyze reliability and assess security which has already exceeded its original category[1],and it becomes the modern management theory. Many facilities and methods have been integrated into one powerful underlying facility. FMEA has been tested well by time as a powerful facility to assess the failure risks of products, process and system[2]. FMEA is adopted firstly in aviation field in 1960s, and has been considered as the basis tool in reliability engineering[3]. When FMEA analysis is applied in mechnical systems, the failure modes that generate severer effects on equipments can be found in time. Based on the failure finding modes, the failure modes can be prevented which can reduce the failure ratio and improve the reliability and stability of equipments[4].

As the FMEA has been applied in various areas and also intercrossed with many subjects, so there emerged some new theories and methods. In the paper [5], FMEA is adopted in the preventing maintenance of digitial-control equipment. Paper [6] makes use of FMEA in absorbing medicine process and reduces the generation ratio of sharp instruments injury. Paper [7] makes use of the FMEA in ship fault information processing system. Through the composite management and analysis of ship equipment fault data, the auxiliary suggestions of fault processing in daily use are proposed. Paper [8] makes use of FMEA and RCM in the maintenance strategy study of heavy-duty vehicle which improves the maintenance quality and management level of maintenance. Paper [9] makes use of process-FMEA in the manufacture process of $T / R$ components and makes process-FMEA and fault analysis integrate more tightly to position fault, they are more accurately and effectively which reduces the effective correction prevention measure. Paper [10] put the FMEA in the fault simulation of some frequency choosing circuit; it is shown that the fault modes with more occurrence ratio and impact results can reduce the work of fault simulation.

\section{Basic Theory of FMEA}

The traditional FMEA analysis method adopts risk priority number (RPN) to various risks according to the size of RPN, which is assumed to prevent and maintain the most dangerous fault under the limited maintenance resource. The size of RPN is equal to the product of fault occurrence probability (O), fault severity (S) and detectable difficulty (D), as known that the three risk factors are changeable [1, 10]. The relative harm effect of every fault could be assessed via RPN value. 
Those faults with higher occurrence probability, more severer extent and more difficult to detect usually have larger RPN value. For those faults with higher RPN, the improving measurement should be researched in reducing fault occurrence probability, fault severity and the detectable difficulty. With the recounted RPN, the fault risk should also be reassessed and resorted until the value of RPN is reduced to the acceptable value [11]. The basic flowchart of FMEA is as shown in Fig. 1.

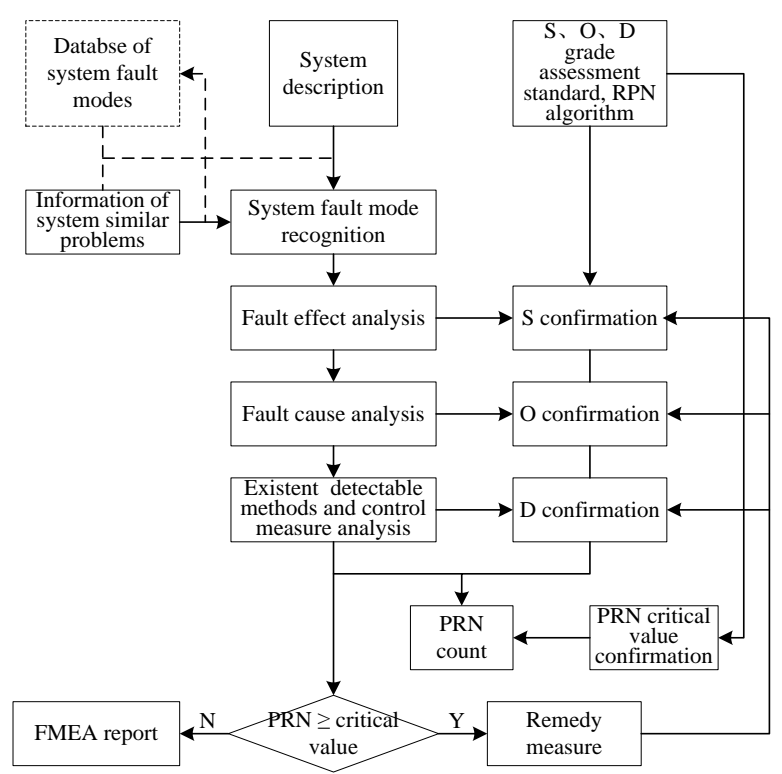

Fig.1 Flowchart of FMEA

From the Fig. 1, it can be found that the risks based on the size of the RPN of potential faults. Through improvement of the fault can avoid the fault happening and reduce their bad effects. The hazards of FMEA could usually be divided into four parts, i.e., the fatal risk, severe risk, medium risk and the smallest risk. Different measures are adopted according to the different grades of risks [12]. The equation used to count RPN of different risk could be written as(1).

$$
\mathrm{RPN}=(\mathrm{S}) \times(\mathrm{O}) \times(\mathrm{D})
$$

In the equation(1), $S$ (severity) represents the evaluation index which is used to evaluate the effect for the next working procedure and system when the potential fault happens. This index could be used only in the outcome of fault and the severity could only be reduced through design. The severity grade can be divided into catastrophic, fateful, critical and low-grade. The values according to these levels are between 1 10; O (occurence) represents the probability of some fault origin or mechanisum appears. The description of frequency degree emphasizes its meaning but not the concrete value. This parameter could also be divided into four standards, very high, high, medium, and low. The values is between 1 10; $\mathrm{D}$ (detectability) is used to measure the difficulty to check out the fault cause which is the index of detecting fault mode, cause, and mechanisum.

\section{Risk and Feasibility Parameter Fuzzy Estimation}

From (1) we can find that, if we want to estimate some fault modes, the three parameters of this fault mode should be quantified firstly and then the three parameters should be multiplied in order to get the RPN of the fault mode. In the paper, we utilize the quantitative method in paper [11] to get the three parameters.

A. Quantitative Estimation of Severity Probability and Detecable feasibitliy

Centroid fuzzy-free method defines the centroid of fuzzy number $\bar{A}$ as its fuzzy-free value. The count equation is as follows [13]:

$\bar{x}_{0}(\bar{A})=\frac{\int_{a}^{d} x u_{\tilde{A}}(x) d x}{\int_{a}^{d} u_{\tilde{A}}(x) d x}$ 
In eqiation (2), $\bar{x}_{0}(\bar{A})$ is the centroid value of fuzzy number $\bar{A} ; u_{\tilde{A}}(x)$ is the subject function of fuzzy number $\bar{A}$. If the fuzzy number $\bar{A}$ is denoted through cut assembly $\alpha$ i.e. $\bar{A}=\cup_{\alpha} \alpha \cdot(A)_{\alpha}=\cup_{\alpha} \alpha \cdot\left[(x)_{\alpha}^{L} \cdot(x)_{\alpha}^{U}\right](0<\alpha \leq 1)$ then the fuzzy -free value could be counted via (2) (4).

$$
\begin{aligned}
\int_{a}^{d} \mu_{\tilde{A}}(x) d x= & \frac{1}{2 n}\left[(x)_{a_{0}}^{U}-(x)_{a_{0}}^{L}\right]+ \\
& \frac{1}{2 n}\left[(x)_{a_{n}}^{U}-(x)_{a_{n}}^{L}\right]+ \\
& \frac{1}{n} \sum_{i=1}^{n-1}\left[(x)_{a_{i}}^{U}-(x)_{a_{i}}^{L}\right] \\
\int_{a}^{d} x \mu_{\tilde{A}}(x) d x= & \frac{1}{6 n}\left[(x)_{a_{0}}^{2 U}-(x)_{a_{0}}^{2 L}\right]+ \\
& \frac{1}{6 n}\left[(x)_{a_{n}}^{2 U}-(x)_{a_{n}}^{2 L}\right]+ \\
& \frac{1}{3 n} \sum_{i=1}^{n-1}\left[(x)_{a_{n}}^{2 U}-(x)_{a_{n}}^{2 L}\right]+ \\
& \frac{1}{6 n} \sum_{i=1}^{n-1}\left[(x)_{a_{i}}^{U} \cdot(x)_{a_{i+1}}^{U}-(x)_{a_{i}}^{L} \cdot(x)_{a_{i+1}}^{L}\right]
\end{aligned}
$$

The paper [11] introduces the basic step of count the three parameters through (2) (4) based on the fuzzy theory. The basic step is as follows:

Step1: The overall occurrence, severity, detectability of every fault and the overall fuzzy weight of S, O, D is counted via (5) (7) based on the opinions of experts.

$$
\begin{aligned}
\tilde{R}_{i j}^{o}= & \sum_{j=1}^{m} k_{j} \tilde{R}_{i j}^{o}=\left(\sum_{j=1}^{m} k_{j} R_{i j L}^{o}, \sum_{j=1}^{m} k_{j} R_{i j M}^{o}, \sum_{j=1}^{m} k_{j} R_{i j U}^{o}\right), \\
& i=1, \cdots \bullet, n \tilde{w}^{o}=\sum_{j=1}^{m} k_{j} \tilde{w}_{j}^{o}= \\
& \left(\sum_{j=1}^{m} k_{j} w_{j L}^{o}, \sum_{j=1}^{m} k_{j} w_{j M}^{o}, \sum_{j=1}^{m} k_{j} w_{j U}^{o}\right) \\
\tilde{R}_{i j}^{S}= & \sum_{j=1}^{m} k_{j} \tilde{R}_{i j}^{S}=\left(\sum_{j=1}^{m} k_{j} R_{i j L}^{S}, \sum_{j=1}^{m} k_{j} R_{i j U}^{S}, \sum_{j=1}^{m} k j R_{i j U}^{S}\right), \\
& i=1, \cdots, n \tilde{w}^{S}=\sum_{j=1}^{m} k_{j} \tilde{w}_{j}^{S} \\
= & \left(\sum_{j=1}^{m} k_{j} w_{j L}^{S}, \sum_{j=1}^{m} k_{j} w_{j M}^{S}, \sum_{j=1}^{m} k_{j} w_{j U}^{S}\right) \\
\tilde{R}_{i j}^{D}= & \sum_{j=1}^{m} k_{j} \tilde{R}_{i j}^{D}=\left(\sum_{j=1}^{m} k_{j} R_{i j L}^{D}, \sum_{j=1}^{m} k_{j} R_{i j M}^{D}, \sum_{j=1}^{m} k_{j} R_{i j U}^{D}\right) \\
& i=1, \cdots, n \tilde{w}^{D}=\sum_{j=1}^{m} k_{j} \tilde{w}_{j}^{D}= \\
( & \left.\sum_{j=1}^{m} k_{j} w_{j L}^{D}, \sum_{j=1}^{m} k_{j} w_{j M}^{D}, \sum_{j=1}^{m} k_{j} w_{j U}^{D}\right)
\end{aligned}
$$

Step 2: The RPN value and cut assembly $\alpha$ of every fault mode is defined through according equations.

Step 3: With the centroid fuzzy-free methods, the fuzzy form of RPN is transformed into precise value. When the unit interval $[0,1]$ is divided equally by different cut assembly $\alpha$, the centric of fuzzy number RPN is confirmed. 
Step 4: Based on the centroid of RPN of every fuzzy risk priority value, the risk order of every fault mode is accomplished by the method in the next sub-section.

B. Sort method for different RPN

In section A, the quantified method of the three parameters of severity, occurrence probability and detectable difficulty in risk priority degree is researched. And based on the method, the RPN of every fault mode is counted out. With this advanced research, the most urgent fault mode that needs to be solved via FMEA is picked out. To solve this problem, [3] puts forward a decision-making method that is called Scree-plot measure. The RPN of every fault mode is sorted out according to the size of its number from the smallest to the biggest. Then the RPN of every fault mode is plotted out in some Fig, the demonstration is showed in Fig. 2.

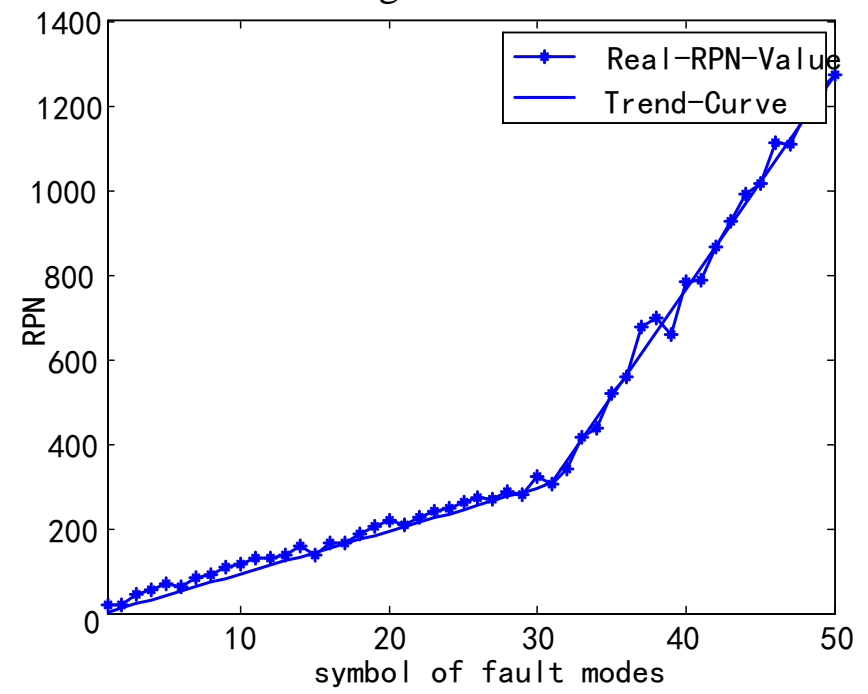

Fig.2 Scree-plot of every RPN

As showed in Fig. 2, real line stands for the RPN of every fault, broken line stands for the different alteration trend of various RPN. In Fig. 2, the alteration trend of the former $30 \mathrm{RPN}$ is not evident which almost belongs to the same smooth area. But the alteration trend of the latter 20 RPN is clear which has a very high slope. The RPN in the latter trend changes from a very small value to a relatively high number in a comparatively slow time. [3] makes this interzone as the RPN that needs to be firstly processed. Then from the biggest number of RPN to the smallest, every different RPN with different fault mode is processed.

C. Quantitative estimation of feasible parameter

When the most urgent RPN that needs to be managed at first time is fixed on, the according method that reduces the value of this RPN has to be chosen out. As showed in (1), if the value of RPN is needed to be reduced, we can decrease $S, O$, and D to reduce RPN. So, there are lots of methods that could reduce RPN of the same fault mode. And how to choose out the most effective method is a very important problem. [3] makes a study on how to choose an effective method from all the usable methods when FMEA is used and puts forward the concept of correction measure feasibility F (F is relative with security, fee, resource, time, quality, reliability and so on). For some concrete fault mode, [3] puts forward that the ratio between the alteration quantitative of RPN before and after the correction method and feasibility parameter is higher, the correction method is better. To solve the problem that how to quantify the feasibility parameter, [14] makes research on the estimation method of this parameter based on the well-known Pareto-priority-index count program. And [15] puts forward a new method that computes the geometric mean value of every factor (e.g. fee, consumed time, success probability) related with feasibility parameter $F$ and treats this value as the concrete value of F. To solve the fuzzy character of expert judge and uncertain problem in traditional FMEA, the dependent linguistic ordered weighted geometric operator is used in FMEA risk estimation.

After the research of the various RPN selective method, the quantitative method based on fuzzy measure for feasibility parameter $\mathrm{F}$ is put forward in this paper. The feasibility parameter $\mathrm{F}$ is 
quantified via fuzzy theory and the ratio between RPN alteration value and feasibility parameter is chosen as the reference to choose the correction method. The concrete account method is showed in $(8)^{[3]}$

$R E F_{\text {_value }}=\frac{R P N_{\text {iBefore }}-R P N i_{\text {After }}}{F_{i}}=\frac{\Delta R P N}{F_{i}}(8)$

In (8), $R P N_{\text {iBefore }}$ stands for the RPN value of some fault mode before the correction. $R P N i_{A f t e r}$ stands for the RPN value of some fault mode after the correction. $\triangle R P N$ stands for the RPN alteration value before and after the correction. $F_{i}$ stands for the feasibility parameter of this fault mode.

The quantitative method of $F_{i}$ could be found out in subsection A. The basic process is almost the same so the it's not discussed in detail here.

\section{Conclusion}

This paper analyses the reliability of aviation electronic sub-system of UAV. Based on the quantified feasibility parameter F, the fuzzy theory and FMEA is combined and is used in the reliability analysis of aviation electronic sub-system. The validity of this method is validated by concrete report table.

\section{References}

[1] D. Yun Hui, H. Zhi Hui, Z. Hai Rong. FMEA Theories and Methods-Review on Research Progress. Quality Safety and Mangement, 2010, 11(2):23-26.

[2] G. Wen De, L. Mu. FMEA used for eliminating puzzles. Quality Safety and Mangement, 2011, 12(3):33-36.

[3] B. Zigmund, G. Pavel, N. Oren. Expanded FMEA(EFMEA). 2004 IEEE RAMS, 2004:31-36.

[4] T. Xu, S. Zhen Yu, S. Pei, et al. Use of FMEA Technology in Army Equipment Maintenance. Value project, 2010, 12(5):324-328.

[5] X. Jun Xiong, Z. Jin Ping, T. Hai Ning, et al. Study on equipment fault management based on FMEA. Machine Tool \& Hydraulics, 2012, 40(7): 184-185,190.

[6] H. Tian Lan, J. Li Fang, L. Li Rong, et al. Study on FMEA and its application in medicine absorption flow. Business Defense, 2010, 12(4):35-36.

[7] Z. Wei Dong. Signal Processing System of Ship Equipment Fault based on FMEA. Computer and modernization, 2012, 12(5):172-175.

[8] Z. Yu Mei. Matainetance maneuver study of heavy car based on FMEA and RCM. Technique Study and exploration, 2012, 12(5):12-18.

[9] D. Jun, H. Hong Ping. Study and application of PFMEA in T/R composite components. Electro-Mechanical Engineering, 2010, 26(6):6-10.

[10]G. Hong Qiang, L. Fei, G. Li. FMEA and its application in the fault simulation of some frequency choice. Engineering Design Journal 2010, 17(2):124-127.

[11]L. Yi Jia, Y. Jian Xing. Quality risk analysis of submarine pipeline in construction period base on FMEA and fuzzy theory. China Safety Science Journal, 2012, 22(1):112-117.

[12]S. Di Li. Feeling FMEA. Reliabitliy and Application, 2010, 15(2):13-16. 
[13]W. Ying Ming, C. Kwai Sang, G. Ka Poon, et al. Risk evaluation in failure mode and effects analysis using fuzzy weighted geometric mean. Expert Systems with Applications, 2009, 36(2):1195-1207.

[14]B. Hartman. Implementing Quality Improvement. The Juran Report, 1983, 2(11):156-159. 